\title{
Canonical Duality for Box Constrained Nonconvex and Nonsmooth Optimization Problems
}

\author{
Jing Liu and Huicheng Liu \\ Department of Mathematics and Computational Sciences, Wuyi University, Jiangmen, Guangdong 529020, China \\ Correspondence should be addressed to Jing Liu; liujingftao@163.com
}

Received 7 May 2015; Accepted 29 June 2015

Academic Editor: Dapeng P. Du

Copyright (C) 2015 J. Liu and H. Liu. This is an open access article distributed under the Creative Commons Attribution License, which permits unrestricted use, distribution, and reproduction in any medium, provided the original work is properly cited.

\begin{abstract}
This paper presents an application of the canonical duality theory for box constrained nonconvex and nonsmooth optimization problems. By use of the canonical dual transformation method, which is developed recently, these very difficult constrained optimization problems in $R^{n}$ can be converted into the canonical dual problems, which can be solved by deterministic methods. The global and local extrema can be identified by the triality theory. Some examples are listed to illustrate the applications of the theory presented in the paper.
\end{abstract}

\section{Primal Problems}

The methods of solving nonconvex and nonsmooth optimization have been the topic of intense research during the last forty years. As we know, the general methods of nonsmooth optimization are based on some subdifferentials. However, in the general nonconvex and nonsmooth optimization problem, due to the nonconvexity and nonsmoothness of the objective functions and inequality constraints, the computation of subdifferential is rather time-consuming. So the traditional theories and direct methods are very difficult in solving the nonconvex and nonsmooth optimization and global optimality with constraints. Recently, some effective methods have been studied to solve certain box constrained nonconvex minimization problems [1-8].

In this paper, the primary goal is to solve the following box constrained nonconvex and nonsmooth optimization problems (in short, the primal problem $(\mathscr{P})$ ). Consider

$$
(\mathscr{P}): \min _{x \in \chi_{a}} P_{s}(x)=\frac{1}{2} x^{T} A x+W(x, s)-x^{T} f,
$$

where $\chi_{a}=\left\{x \in R^{n} \mid-1 \leq x \leq 1, x \in\{-1,1\}^{n}\right\}$ (the notation $\{-1,1\}^{n}$ denotes integer vectors of $R^{n}$ with components either -1 or 1$)$ is a feasible space, $f \in R^{n}$ is a nonzero vector, and
$A=A^{T}$ is an $n$ times $n$ matrix. $W(x, s): R^{n} \times R^{m} \rightarrow R$ is a nonconvex and nonsmooth function. Here, we simply assume that $W(x, s)$ is defined by

$$
\begin{aligned}
& W(x, s) \\
& \quad= \begin{cases}\frac{1}{2} a_{1}\left(\frac{1}{2}|B x-s|^{2}-\mu_{1}\right)^{2}, & \text { if }|B x-s| \leq c, \\
\frac{1}{2} a_{2}\left(\frac{1}{2}|B x-s|^{2}-\mu_{2}\right)^{2}, & \text { if }|B x-s|>c,\end{cases}
\end{aligned}
$$

where $B$ is an $m$ times $n$ matrix, $s \in R^{m}$ is a vector of parameter, $\mu_{2}=c^{2} / 2+\sqrt{a_{1} / a_{2}}\left|c^{2} / 2-\mu_{1}\right|, \mu_{1}$ is an arbitrary constant, $a_{1}$ and $a_{2}$ are both nonzero positive constants, $|\cdot|: R^{n} \rightarrow$ $R$ is the Euclidean norm, and $c$ is an arbitrary positive constant. In constrained global optimization problems, $W$ could be the indicator of a feasible set [9]. In particular, if we abandon the box constraint, problem $(\mathscr{P})$ will be turned into unconstrained optimal problem which has been discussed in [10]. The primal problem $(\mathscr{P})$ appears frequently in many applications, such as semilinear nonconvex partial differential equations, structural limit analysis, discretized optimal control problems with distributed parameters, and network communication $[6,11,12]$. 
In the primal problem $(\mathscr{P})$, the box constraint $-1 \leq x \leq$ 1 is equivalent to $x_{i}^{2} \leq 1(i=1, \ldots, n)$. Thus, based on the traditional Lagrangian multiplier method, we have

$$
L\left(x, \lambda_{i}\right)=\frac{1}{2} x^{T} A x+W(x, s)-x^{T} f+\lambda_{i}\left(x_{i}^{2}-1\right) .
$$

In the case where $A$ is positive definite, for a given $\lambda_{i}>0$ ( $i=$ $1, \ldots, n)$, the traditional dual function can be defined via the Fenchel-Moreau-Young duality theory:

$$
P^{*}\left(\lambda_{i}\right)=\min \left\{L\left(x, \lambda_{i}\right): x \in R^{n}\right\} \text {. }
$$

However, due to the nonconvexity of the objective function $P_{s}(x)$, the Young-Fenchel inequality can lead to a weak duality relationship in general nonconvex systems:

$$
\min _{x \in \chi_{a}} P_{s}(x) \geq \max _{\lambda_{i} \geq 0} P^{*}\left(\lambda_{i}\right)
$$

The nonzero value $\theta=\min _{x \in \chi_{a}} P_{s}(x)-\max _{\lambda_{i} \geq 0} P^{*}\left(\lambda_{i}\right)$ is called the duality gap, which is usually $-\infty$ if $A$ is indefinite. This nonzero duality gap shows that the Fenchel-Rockafellar duality theory and method can be used mainly in convex systems. In order to eliminate this duality gap, many modified Fenchel-Rockafellar duality theories and methods for nonconvex optimization problems have been proposed [1220]. Recently, the so-called canonical dual transformation method (without the duality gap) has been developed in general nonconvex systems [21]. This method is a newly useful tool for optimal problem. At present, the method has been successfully used for a large class of nonsmooth or nonconvex minimization $[9,10,21,22]$.

In this paper, we will present the application of the canonical dual transformation method for the solutions of the box constrained nonconvex and nonsmooth optimization problems $(\mathscr{P})$ in $R^{n}$. In the next section, a perfect dual problem is formulated, which is equivalent to the primal problem in the sense that they have the same set of critical points. Section 3 shows the sufficient conditions for global and local minima. In Section 4, some concrete examples for box constrained nonconvex and nonsmooth optimization problems are presented. We state some conclusions in Section 5 .

\section{Canonical Dual Problem and Complete Solutions}

In order to use the canonical dual transformation method to solve the box constrained nonconvex and nonsmooth optimization problem, we need to reformulate the constraint $-1 \leq x \leq 1$ in canonical form $x_{i}^{2} \leq 1, i=1,2, \ldots, n$.

Following the stand procedure of the canonical dual transformation method developed in [21], the canonical geometrical operator $\Lambda: R^{n} \rightarrow R \times R^{n}$ in the primal problem $(\mathscr{P})$ can be defined as

$$
y=\Lambda(x)=\left(\frac{1}{2}|B x-s|^{2}, x_{i}^{2}-1\right)=(\epsilon, \rho),
$$

where $\epsilon=(1 / 2)|B x-s|^{2}$ is a scale and $\rho=\left\{\rho_{i}\right\}=\left\{x_{i}^{2}-1\right\}$ for $i=1,2, \ldots, n$. Let $\mathscr{Y}_{a} \subset R \times R^{n}$ be the range of the mapping $y=\Lambda(x)$ which can be written as

$$
\mathscr{Y}_{a}=\left\{y=(\epsilon, \rho)^{T} \in R \times R^{n} \mid \epsilon \geq 0, \rho \leq 0\right\} .
$$

According to the canonical transformation method, we define a real-valued function $V: \mathscr{Y}_{a} \rightarrow R$. Thus, the nonconvex and nonsmooth function $W(x, s)$ can be written in a canonical form:

$$
W(x, s)=V(y) .
$$

Then $V(y)$ is a canonical function defined on the subset $\mathscr{Y}_{a} \subset$ $R$ which can be written as

$$
\begin{aligned}
V(y) & =V(\epsilon, \rho) \\
& = \begin{cases}\frac{1}{2} a_{1}\left(\epsilon-\mu_{1}\right)^{2}+\varphi(\rho), & \text { if } 0 \leq \epsilon \leq \frac{c^{2}}{2}, \\
\frac{1}{2} a_{2}\left(\epsilon-\mu_{2}\right)^{2}+\varphi(\rho), & \text { if } \epsilon>\frac{c^{2}}{2},\end{cases}
\end{aligned}
$$

where

$$
\varphi(\rho)= \begin{cases}0, & \text { if } \rho \leq 0 \\ +\infty, & \text { otherwise }\end{cases}
$$

We yet assume that matrix $A$ is invertible. For each given nonzero vector $f \in R^{n}$, the function $F: R^{n} \rightarrow R$ is defined by

$$
F(x)=x^{T} f-\frac{1}{2} x^{T} A x,
$$

where $F$ is a canonical function on $R^{n}$ since its Gâteaux derivative $x^{*}=D F(x)=f-A x: R^{n} \rightarrow R^{n}$ is one-to-one onto mapping. Thus, we can rewrite the primal minimization problem $(\mathscr{P})$ in the unconstrained canonical form $\left(\left(\mathscr{P}_{q}\right)\right.$ in short):

$$
\left(\mathscr{P}_{q}\right): P(x)=V(\Lambda(x))-F(x) \longrightarrow \text { sta, } \quad \forall x \in R^{n},
$$

where sta stands for finding all the stationary points of $P(x)$.

Let $y^{*}=\left(\epsilon^{*}, \rho^{*}\right)^{T} \in R \times R^{n}$ be a dual variable of $y$ and $A+B^{T} B \epsilon^{*}+2 \operatorname{Diag} \rho_{i}^{*} \neq 0$, where $\operatorname{Diag} \rho_{i}^{*} \in R^{n \times n}$ represents a diagonal matrix with $\rho_{i}^{*}(i=1,2, \ldots, n)$ as its diagonal entries and the dual variable $\rho^{*}=\left\{\rho_{i}^{*}\right\}(i=1,2, \ldots, n)$ is also a vector in $R^{n}$. Then the $\Lambda$-canonical conjugate $F^{\Lambda}\left(y^{*}\right)$ of the canonical function $F(x)$ can be well defined by the $\Lambda$ canonical dual transformation [21]:

$$
\begin{gathered}
F^{\Lambda}\left(y^{*}\right)=\left\{\left\langle\Lambda(x), y^{*}\right\rangle-F(x) \mid D F(x)\right. \\
\left.=\left\langle D \Lambda(x), y^{*}\right\rangle, x \in X_{a}\right\}=-\frac{1}{2}\left(G\left(\epsilon^{*}\right)\right)^{T} \\
\cdot\left(F\left(\epsilon^{*}, \rho^{*}\right)\right)^{-1} G\left(\epsilon^{*}\right)+\frac{1}{2} s^{T} s \epsilon^{*}-\sum_{i=1}^{n} \rho_{i}^{*},
\end{gathered}
$$


where $F\left(\epsilon^{*}, \rho^{*}\right) \stackrel{\text { def }}{=} A+B^{T} B \epsilon^{*}+2 \operatorname{Diag} \rho_{i}^{*}, G\left(\epsilon^{*}\right) \stackrel{\text { def }}{=} f+B^{T} s \epsilon^{*}$, $D \Lambda(x)$ is the Gâteaux derivative of $\Lambda$ at $x$, and $D F(x)$ denotes the gradient $F$ at $x$.

In the case of $a_{1}>a_{2}$, by the definition of the canonical function, $V: y_{a} \rightarrow R$ is Gâteaux differentiable, and the duality relation $y^{*}=D V(y): \mathscr{Y}_{a} \rightarrow \mathscr{Y}_{a}^{*}=R \times R^{n}$ is invertible, where $D V$ denotes the Gâteaux derivative of $V$ at $y$. Thus, the canonical conjugate $V^{*}\left(y^{*}\right)=V^{*}\left(\epsilon^{*}, \rho^{*}\right)$, where $y^{*}=\left(\epsilon^{*}, \rho^{*}\right)^{T} \in R \times R^{n}$ of $V(y)$, can be obtained by the Legendre transformation:

$$
\begin{aligned}
V^{*}\left(y^{*}\right) & =\left\{\left\langle y, y^{*}\right\rangle-V(y) \mid D V(y)=y^{*}\right\} \\
& = \begin{cases}\frac{1}{2 a_{1}}\left(\epsilon^{*}\right)^{2}+\mu_{1} \epsilon^{*}, & \text { if }-a_{1} \mu_{1} \leq \epsilon^{*} \leq a_{1}\left(\frac{c^{2}}{2}-\mu_{1}\right), \rho^{*} \geq 0 \\
\frac{c^{2}}{2} \epsilon^{*}-\frac{1}{2} a_{1}\left(\frac{c^{2}}{2}-\mu_{1}\right)^{2}, & \text { if } a_{1}\left(\frac{c^{2}}{2}-\mu_{1}\right)<\epsilon^{*} \leq a_{2}\left(\frac{c^{2}}{2}-\mu_{2}\right), \rho^{*} \geq 0 \\
\frac{1}{2 a_{2}}\left(\epsilon^{*}\right)^{2}+\mu_{2} \epsilon^{*}, & \text { if } \epsilon^{*}>a_{2}\left(\frac{c^{2}}{2}-\mu_{2}\right), \rho^{*} \geq 0 .\end{cases}
\end{aligned}
$$

The dual feasible spaces are three subsets of $R \times R^{n}$ :

$$
\begin{aligned}
& \mathscr{Y}_{\alpha_{1}}^{*}=\left\{y^{*}=\left(\epsilon^{*}, \rho^{*}\right)^{T} \in R \times R^{n} \mid-a_{1} \mu_{1} \leq \epsilon^{*}\right. \\
& \leq\left.a_{1}\left(\frac{c^{2}}{2}-\mu_{1}\right), \rho^{*} \geq 0, \operatorname{det} F\left(\epsilon^{*}, \rho^{*}\right) \neq 0\right\}, \\
& \mathscr{Y}_{\beta_{1}}^{*}=\left\{y^{*}=\left(\epsilon^{*}, \rho^{*}\right)^{T} \in R \times R^{n} \mid a_{1}\left(\frac{c^{2}}{2}-\mu_{1}\right)<\epsilon^{*}\right. \\
&\left.\leq a_{2}\left(\frac{c^{2}}{2}-\mu_{2}\right), \rho^{*} \geq 0, \operatorname{det} F\left(\epsilon^{*}, \rho^{*}\right) \neq 0\right\}, \\
& \mathscr{Y}_{\gamma_{1}}^{*}=\left\{y^{*}=\left(\epsilon^{*}, \rho^{*}\right)^{T} \in R \times R^{n} \mid \epsilon^{*}\right. \\
&\left.>a_{2}\left(\frac{c^{2}}{2}-\mu_{2}\right), \rho^{*} \geq 0, \operatorname{det} F\left(\epsilon^{*}, \rho^{*}\right) \neq 0\right\} .
\end{aligned}
$$

Thus, on the dual feasible spaces, the canonical dual function $P^{d}\left(y^{*}\right)$ can be formulated as

$$
\begin{aligned}
P^{d}\left(y^{*}\right) & =\operatorname{sta}\left\{\Xi\left(x, y^{*}\right): x \in R^{n}\right\} \\
& =F^{\Lambda}\left(y^{*}\right)-V^{*}\left(y^{*}\right) .
\end{aligned}
$$

The canonical dual problem can be formulated as problem $\left(\mathscr{P}_{q}^{d}\right)$ :

$$
\begin{aligned}
& P^{d}\left(y^{*}\right)=P^{d}\left(\epsilon^{*}, \rho^{*}\right) \\
& = \begin{cases}-\frac{1}{2}\left(G\left(\epsilon^{*}\right)\right)^{T}\left(F\left(\epsilon^{*}, \rho^{*}\right)\right)^{-1} G\left(\epsilon^{*}\right)+\frac{1}{2} s^{T} s \epsilon^{*}-\left(\frac{1}{2 a_{1}}\left(\epsilon^{*}\right)^{2}+\mu_{1} \epsilon^{*}\right)-\sum_{i=1}^{n} \rho_{i}^{*}, & \text { if }\left(\epsilon^{*}, \rho^{*}\right)^{T} \in \mathscr{Y}_{\alpha_{1}}^{*}, \\
-\frac{1}{2}\left(G\left(\epsilon^{*}\right)\right)^{T}\left(F\left(\epsilon^{*}, \rho^{*}\right)\right)^{-1} G\left(\epsilon^{*}\right)+\frac{1}{2} s^{T} s \epsilon^{*}-\left(\frac{c^{2}}{2} \epsilon^{*}-\frac{1}{2} a_{1}\left(\frac{c^{2}}{2}-\mu_{1}\right)^{2}\right)-\sum_{i=1}^{n} \rho_{i}^{*}, & \text { if }\left(\epsilon^{*}, \rho^{*}\right)^{T} \in \mathscr{Y}_{\beta_{1}}^{*}, \\
-\frac{1}{2}\left(G\left(\epsilon^{*}\right)\right)^{T}\left(F\left(\epsilon^{*}, \rho^{*}\right)\right)^{-1} G\left(\epsilon^{*}\right)+\frac{1}{2} s^{T} s \epsilon^{*}-\left(\frac{1}{2 a_{2}}\left(\epsilon^{*}\right)^{2}+\mu_{2} \epsilon^{*}\right)-\sum_{i=1}^{n} \rho_{i}^{*}, & \text { if }\left(\epsilon^{*}, \rho^{*}\right)^{T} \in \mathcal{Y}_{\gamma_{1}}^{*} .\end{cases}
\end{aligned}
$$


In the case of $a_{1}<a_{2}$, the canonical conjugate $V^{*}\left(y^{*}\right)=$ $V^{*}\left(\epsilon^{*}, \rho^{*}\right)$ of $V(y)$ can be written as

$$
\begin{aligned}
V^{*}\left(y^{*}\right)= & \left\{\left\langle y, y^{*}\right\rangle-V(y) \mid D V(y)=y^{*}\right\} \\
= & \begin{cases}\frac{1}{2 a_{2}}\left(\epsilon^{*}\right)^{2}+\mu_{2} \epsilon^{*}, & \text { if }-a_{2} \mu_{2} \leq \epsilon^{*} \leq a_{2}\left(\frac{c^{2}}{2}-\mu_{2}\right), \rho^{*} \geq 0 . \\
\frac{c^{2}}{2} \epsilon^{*}-\frac{1}{2} a_{2}\left(\frac{c^{2}}{2}-\mu_{2}\right)^{2}, & \text { if } a_{2}\left(\frac{c^{2}}{2}-\mu_{2}\right)<\epsilon^{*} \leq a_{1}\left(\frac{c^{2}}{2}-\mu_{1}\right), \rho^{*} \geq 0 . \\
\frac{1}{2 a_{1}}\left(\epsilon^{*}\right)^{2}+\mu_{1} \epsilon^{*}, & \text { if } \epsilon^{*}>a_{1}\left(\frac{c^{2}}{2}-\mu_{1}\right), \rho^{*} \geq 0 .\end{cases}
\end{aligned}
$$

It is similar to that in the case of $a_{1}>a_{2}$ where the dual feasible spaces are also three subsets of $R \times R^{n}$ :

$$
\begin{aligned}
& \mathscr{Y}_{\alpha_{2}}^{*}=\left\{y^{*}=\left(\epsilon^{*}, \rho^{*}\right)^{T} \in R \times R^{n} \mid-a_{2} \mu_{2} \leq \epsilon^{*}\right. \\
& \left.\leq a_{2}\left(\frac{c^{2}}{2}-\mu_{2}\right), \rho^{*} \geq 0, \operatorname{det} F\left(\epsilon^{*}, \rho^{*}\right) \neq 0\right\}, \\
& \mathscr{Y}_{\beta_{2}}^{*}=\left\{y^{*}=\left(\epsilon^{*}, \rho^{*}\right)^{T} \in R \times R^{n} \mid a_{2}\left(\frac{c^{2}}{2}-\mu_{2}\right)<\epsilon^{*}\right.
\end{aligned}
$$$$
P^{d}\left(y^{*}\right)=P^{d}\left(\epsilon^{*}, \rho^{*}\right)
$$$$
= \begin{cases}-\frac{1}{2}\left(G\left(\epsilon^{*}\right)\right)^{T}\left(F\left(\epsilon^{*}, \rho^{*}\right)\right)^{-1} G\left(\epsilon^{*}\right)+\frac{1}{2} s^{T} s \epsilon^{*}-\left(\frac{1}{2 a_{2}}\left(\epsilon^{*}\right)^{2}+\mu_{2} \epsilon^{*}\right)-\sum_{i=1}^{n} \rho_{i}^{*}, & \text { if }\left(\epsilon^{*}, \rho^{*}\right)^{T} \in \mathcal{Y}_{\alpha_{2}}^{*}, \\ -\frac{1}{2}\left(G\left(\epsilon^{*}\right)\right)^{T}\left(F\left(\epsilon^{*}, \rho^{*}\right)\right)^{-1} G\left(\epsilon^{*}\right)+\frac{1}{2} s^{T} s \epsilon^{*}-\left(\frac{c^{2}}{2} \epsilon^{*}-\frac{1}{2} a_{2}\left(\frac{c^{2}}{2}-\mu_{2}\right)^{2}\right)-\sum_{i=1}^{n} \rho_{i}^{*}, & \text { if }\left(\epsilon^{*}, \rho^{*}\right)^{T} \epsilon \mathcal{Y}_{\beta_{2}}^{*}, \\ -\frac{1}{2}\left(G\left(\epsilon^{*}\right)\right)^{T}\left(F\left(\epsilon^{*}, \rho^{*}\right)\right)^{-1} G\left(\epsilon^{*}\right)+\frac{1}{2} s^{T} s \epsilon^{*}-\left(\frac{1}{2 a_{1}}\left(\epsilon^{*}\right)^{2}+\mu_{1} \epsilon^{*}\right)-\sum_{i=1}^{n} \rho_{i}^{*}, & \text { if }\left(\epsilon^{*}, \rho^{*}\right)^{T} \in \mathcal{Y}_{\gamma_{2}}^{*},\end{cases}
$$

Theorem 1 (perfect duality theorem). Suppose that vector $\bar{y}^{*}=\left(\bar{\epsilon}^{*}, \bar{\rho}^{*}\right)^{T}$ is a stationary point of the canonical dual problem $\left(\mathscr{P}_{q}^{d}\right)$; then the vector defined by

$$
\bar{x}=F\left(\bar{\epsilon}^{*}, \bar{\rho}^{*}\right)^{-1} G\left(\bar{\epsilon}^{*}\right)
$$

is a stationary point of the primal problem $\left(\mathscr{P}_{q}\right)$, and

$$
P(\bar{x})=P^{d}\left(\bar{\epsilon}^{*}, \bar{\rho}^{*}\right) .
$$

$$
\begin{aligned}
& \leq\left.a_{1}\left(\frac{c^{2}}{2}-\mu_{1}\right), \rho^{*} \geq 0, \operatorname{det} F\left(\epsilon^{*}, \rho^{*}\right) \neq 0\right\}, \\
& \mathcal{Y}_{\gamma_{2}}^{*}=\left\{y^{*}=\left(\epsilon^{*}, \rho^{*}\right)^{T} \in R \times R^{n} \mid \epsilon^{*}\right. \\
&\left.>a_{1}\left(\frac{c^{2}}{2}-\mu_{1}\right), \rho^{*} \geq 0, \operatorname{det} F\left(\epsilon^{*}, \rho^{*}\right) \neq 0\right\} .
\end{aligned}
$$

Hence, on the dual feasible spaces, the canonical dual problem $\left(\mathscr{P}_{q}^{d}\right)$ can be formulated as 
In terms of $\bar{x}=F\left(\bar{\epsilon}^{*}, \bar{\rho}^{*}\right)^{-1} G\left(\bar{\epsilon}^{*}\right)$ and (24), one has that

$$
\frac{1}{a_{1}} \bar{\epsilon}^{*}+\mu_{1}=\frac{1}{2}|B x-s|^{2}=\epsilon
$$

By the condition $0 \leq \epsilon \leq c^{2} / 2$, it is obtained that $-a_{1} \mu_{1} \leq$ $\epsilon^{*} \leq a_{1}\left(c^{2} / 2-\mu_{1}\right)$. On the other hand, as $\bar{y}^{*}=\left(\bar{\epsilon}^{*}, \bar{\rho}^{*}\right)^{T}$ is the stationary point of $\left(\mathscr{P}_{q}^{d}\right)$, then

$$
\begin{gathered}
\bar{x}_{i}^{2}\left(\bar{\epsilon}^{*}, \bar{\rho}^{*}\right)-1 \leq 0, \quad \bar{\rho}_{i}^{*} \geq 0, \\
\bar{\rho}_{i}^{*}\left(\bar{x}_{i}^{2}\left(\bar{\epsilon}^{*}, \bar{\rho}^{*}\right)-1\right)=0, \quad i=1, \ldots, n,
\end{gathered}
$$

where $\bar{x}_{i}\left(\bar{\epsilon}^{*}, \bar{\rho}^{*}\right)$ is the $i$ th component of the vector $\bar{x}=$ $F\left(\bar{\epsilon}^{*}, \bar{\rho}^{*}\right)^{-1} G\left(\bar{\epsilon}^{*}\right)$. It is obvious that $\bar{x}\left(\bar{\epsilon}^{*}, \bar{\rho}^{*}\right)$ is also a stationary point of the primal problem $(\mathscr{P})$. Thus, by $\bar{x}=$ $F\left(\bar{\epsilon}^{*}, \bar{\rho}^{*}\right)^{-1} G\left(\bar{\epsilon}^{*}\right)$ and

$$
\begin{aligned}
\frac{1}{2 a_{1}}\left(\bar{\epsilon}^{*}\right)^{2}+\mu_{1} \bar{\epsilon}^{*}= & -\frac{1}{2} a_{1}\left(\frac{1}{2}|B \bar{x}-s|^{2}-\mu_{1}\right)^{2} \\
& +\frac{1}{2}|B \bar{x}-s|^{2} \bar{\epsilon}^{*},
\end{aligned}
$$

we have

$$
\begin{aligned}
P^{d}\left(\bar{\epsilon}^{*}, \bar{\rho}^{*}\right)= & \frac{1}{2} x^{T} F\left(\bar{\epsilon}^{*}, \bar{\rho}^{*}\right) x-s^{T} B \bar{\epsilon}^{*} x+\frac{1}{2} s^{T} s \\
& -x^{T} f \\
= & \frac{1}{2}|B \bar{x}-s|^{2} \bar{\epsilon}^{*}+\bar{\rho}_{i}^{*}\left(\bar{x}_{i}^{2}\left(\bar{\epsilon}^{*}, \bar{\rho}^{*}\right)-1\right) \\
& +\frac{1}{2} x^{T} A x-x^{T} f \\
& +\frac{1}{2} a_{1}\left(\frac{1}{2}|B \bar{x}-s|^{2}-\mu_{1}\right)^{2} \\
& -\frac{1}{2}|B \bar{x}-s|^{2} \bar{\epsilon}^{*} \\
= & \frac{1}{2} x^{T} A x+\frac{1}{2} a_{1}\left(\frac{1}{2}|B \bar{x}-s|^{2}-\mu_{1}\right)^{2} \\
& -x^{T} f=P_{s}(\bar{x}) .
\end{aligned}
$$

This proves the theorem.

Theorem 1 shows that, by use of the canonical dual transformation, the primal problem $(\mathscr{P})$ can be converted into a canonical dual problem, which can be solved to obtain the stationary points. However, the stationary conditions are only necessary for nonconvex optimization problem. In the next section we will present the optimality criteria.

\section{Optimality Criteria}

The two subsets of the set $\mathscr{Y}_{\mu}^{*}\left(\mu \in\left\{\alpha_{1}, \beta_{1}, \gamma_{1}, \alpha_{2}, \beta_{2}, \gamma_{2}\right\}\right)$ are, respectively, defined by

$$
\begin{aligned}
& \mathcal{Y}_{+}^{*} \\
& =\left\{\left(\epsilon^{*}, \rho^{*}\right)^{T} \in \mathcal{Y}_{\mu}^{*} \mid F\left(\epsilon^{*}, \rho^{*}\right) \text { is positive definite }\right\}, \\
& y_{-}^{*} \\
& =\left\{\left(\epsilon^{*}, \rho^{*}\right)^{T} \in \mathcal{Y}_{\mu}^{*} \mid F\left(\epsilon^{*}, \rho^{*}\right) \text { is negative definite }\right\} .
\end{aligned}
$$

Theorem 2 (triality theorem). For each dual solution $\bar{y}^{*}=$ $\left(\epsilon^{*}, \rho^{*}\right)^{T} \in \mathscr{Y}_{\mu}^{*}\left(\mu \in\left\{\alpha_{1}, \beta_{1}, \gamma_{1}, \alpha_{2}, \beta_{2}, \gamma_{2}\right\}\right)$, one lets

$$
\bar{x}=\left(F\left(\epsilon^{*}, \rho^{*}\right)\right)^{-1} G(\epsilon) \text {. }
$$

If $\bar{y}^{*}=\left(\epsilon^{*}, \rho^{*}\right)^{T} \in \mathcal{Y}_{+}^{*}$, then $\bar{y}^{*}$ is a global maximizer of $P^{d}$ on $\mathscr{Y}_{+}^{*}$, while $\bar{x}$ is a global minimizer of $P$ on $R^{n}$, and

$$
P(\bar{x})=\min _{x \in \mathscr{X}_{a}} P(x)=\max _{y^{*} \in \mathscr{Y}_{+}^{*}} P^{d}\left(y^{*}\right)=P^{d}\left(\bar{y}^{*}\right) .
$$

If $\bar{y}^{*}=\left(\epsilon^{*}, \rho^{*}\right)^{T} \in \mathcal{Y}_{-}^{*}$, then, on the neighborhoods $\mathscr{Y}_{0}^{*} \subset$ $\mathcal{Y}_{\mu}^{*}$ and $\mathscr{X}_{o} \subset \mathscr{X}_{a}$ of $\bar{y}^{*}$ and the associated $\bar{x}$, respectively, we have that either

$$
P(\bar{x})=\min _{x \in \mathscr{X}_{o}} P(x)=\min _{y^{*} \in \mathscr{Y}_{o}^{*}} P^{d}\left(y^{*}\right)=P^{d}\left(\bar{y}^{*}\right)
$$

or

$$
P(\bar{x})=\max _{x \in \mathscr{X}_{o}} P(x)=\max _{y^{*} \in \mathscr{Y}_{o}^{*}} P^{d}\left(y^{*}\right)=P^{d}\left(\bar{y}^{*}\right)
$$

holds.

Proof. Without loss of generality, we suppose that $\mu=\alpha_{1}$; that is, $\bar{y}^{*}=\left(\bar{\epsilon}^{*}, \bar{\rho}^{*}\right)^{T} \in \mathscr{Y}_{\alpha_{1}}$. The extended Lagrangian $\Xi$ : $R^{n} \times Y_{+}^{*} \rightarrow R$ can be written as

$$
\begin{aligned}
\Xi\left(x, y^{*}\right)= & \Xi\left(x, \epsilon^{*}, \rho^{*}\right) \\
= & (\Lambda(x))^{T} y^{*}-V^{*}\left(y^{*}\right)+\frac{1}{2} x^{T} A x-x^{T} f \\
= & \frac{1}{2} x^{T} F\left(\bar{\epsilon}^{*}, \bar{\rho}^{*}\right) x-s^{T} B \bar{\epsilon}^{*} x+\frac{1}{2} s^{T} s-x^{T} f \\
& -V^{*}\left(y^{*}\right) .
\end{aligned}
$$

It can be easily proved that the critical points of $\Xi\left(x, \epsilon^{*}, \rho^{*}\right)$ solve the primal problem, and $P(\bar{x})=\Xi\left(\bar{x}, \bar{\epsilon}^{*}, \bar{\rho}^{*}\right)$. By Theorem 1, we know that the vector $\bar{y}^{*}=\left(\bar{\epsilon}^{*}, \bar{\rho}^{*}\right)^{T} \in \mathcal{Y}_{\mu}^{*}$ is a stationary point of problem $\left(\mathscr{P}_{q}^{d}\right)$ if and only if $\bar{x}=$ $F\left(\bar{\epsilon}^{*}, \bar{\rho}^{*}\right)^{-1} G\left(\bar{\epsilon}^{*}\right)$ is a stationary point of problem $\left(\mathscr{P}_{q}\right)$ and

$$
P(\bar{x})=\Xi\left(\bar{x}, \bar{y}^{*}\right)=\Xi\left(\bar{x}, \bar{\epsilon}^{*}, \bar{\rho}^{*}\right)=P^{d}\left(\bar{y}^{*}\right) .
$$

If $F\left(\epsilon^{*}, \rho^{*}\right)$ is definite, the extended Lagrangian $\Xi$ is convex on $x \in R^{n}$ and concave in each $\epsilon^{*} \in R$ and $\rho^{*} \in R^{n}$; that is, the extended Lagrangian $\Xi$ is a saddle function on $R^{n} \times \mathscr{Y}_{+}^{*}$. By formula (12) and the definition of $\Lambda(x)$, one has that

$$
\begin{aligned}
V(\Lambda(x)) & =\max _{y^{*} \in \mathscr{Y}_{+}^{*}}\left\{(\Lambda(x))^{T} y^{*}-V^{*}\left(y^{*}\right)\right\} \\
& = \begin{cases}\frac{1}{2}\left(\frac{1}{2}|B x-s|^{2}-\mu_{1}\right)^{2} & \text { if } x \in \mathscr{X}_{a} \\
\infty & \text { otherwise. }\end{cases}
\end{aligned}
$$


Thus, we have

$$
\begin{aligned}
& P^{d}\left(\bar{y}^{*}\right)=\max _{y^{*} \in \mathscr{Y}_{+}^{*}} P^{d}\left(y^{*}\right)=\max _{y^{*} \in \mathscr{Y}_{+}^{*}} \min _{x \in \mathscr{X}_{a}} \Xi\left(x, y^{*}\right) \\
& =\min _{x \in \mathscr{X}_{a}} \max _{y^{*} \in \mathscr{Y}_{+}^{*}} \Xi\left(x, y^{*}\right)=\min _{x \in \mathscr{X}_{a}}\left\{\frac{1}{2} x^{T} A x-x^{T} f\right. \\
& \left.\quad+\max _{y^{*} \in \mathscr{Y}_{+}^{*}}\left\{(\Lambda(x))^{T} y^{*}-\bar{V}^{*}\left(y^{*}\right)\right\}\right\}=\min _{x \in \mathscr{X}_{a}} P_{s}(x) \\
& =P_{s}(\bar{x}) .
\end{aligned}
$$

If $\bar{y}^{*} \in \mathcal{Y}_{-}^{*}$, the matrix $F\left(\epsilon^{*}, \rho^{*}\right)$ is indefinite; the function $\Xi$ is a so-called super-Lagrangian [22]; that is, it is locally concave in each of its variables $x$ and $y^{*}$ on the neighborhood $\mathscr{X}_{o} \times \mathcal{Y}_{o}^{*}$. In this case, by the triality theory developed in [21], we have either

$$
\begin{aligned}
P(\bar{x}) & =\min _{x \in \mathscr{X}_{o}} P(x)=\min _{x \in \mathscr{X}_{o}} \max _{y^{*} \in \mathcal{Y}_{o}^{*}} \Xi\left(x, y^{*}\right) \\
& =\min _{y^{*} \in \mathscr{Y}_{o}^{*}} \max _{x \in \mathscr{X}_{o}} \Xi\left(x, y^{*}\right)=\min _{y^{*} \in \mathscr{Y}_{o}^{*}} P^{d}\left(y^{*}\right) \\
& =P^{d}\left(\bar{y}^{*}\right)
\end{aligned}
$$

or

$$
\begin{aligned}
P(\bar{x}) & =\max _{x \in \mathscr{X}_{o}} P(x)=\max _{x \in \mathscr{X}_{o}} \max _{y^{*} \in \mathscr{Y}_{o}^{*}} \Xi\left(x, y^{*}\right) \\
& =\max _{y^{*} \in \mathscr{Y}_{o}^{*}} \max _{x \in \mathscr{X}_{o}} \Xi\left(x, y^{*}\right)=\max _{y^{*} \in \mathscr{Y}_{o}^{*}} P^{d}\left(y^{*}\right) \\
& =P^{d}\left(\bar{y}^{*}\right) .
\end{aligned}
$$

The theorem is proved.
Theorem 2 shows that if $\bar{y}^{*} \in \mathcal{Y}_{+}^{*}$, then $\bar{x}$ is global minimizer of $P_{s}(x)$, while the stationary point $\bar{y}^{*} \in \mathcal{Y}_{-}^{*}$, then $\bar{x}$ is either a local minimizer or a local maximizer of the primal problem $(\mathscr{P})$. The optimality criteria provides the sufficient conditions of both global and local minina, which can be used to develop for solving the nonconvex and nonsmooth problem with box constraints.

\section{Numerical Tests}

The canonical duality theory can be applied to solve many constrained nonconvex and nonsmooth problems in engineering and science. Now we list some examples to illustrate the applications of the theory presented in this paper.

Example 1. In the case of $n=1$, the nonconvex and nonsmooth function

$$
\begin{gathered}
P(x)=\frac{1}{2} a x^{2}+W(x, s)-f x \\
\text { s.t. } \quad-1 \leq x \leq 1,
\end{gathered}
$$

where

$$
\begin{gathered}
W(x, s)=V(y)= \begin{cases}\frac{1}{2} a_{1}\left(y-\mu_{1}\right)^{2}, & \text { if } 0 \leq y \leq \frac{c^{2}}{2}, \\
\frac{1}{2} a_{2}\left(y-\mu_{2}\right)^{2}, & \text { if } y>\frac{c^{2}}{2},\end{cases} \\
y=\Lambda(x)=\frac{1}{2}(b x-s)^{2} .
\end{gathered}
$$

If we choose $a=-0.5, b=-1.0, f=1 / 6, s=1 / 4,20=$ $a_{1}>a_{2}=5, \mu_{1}=1 / 4, c=1 / 2$, then $\mu_{2}=3 / 8$; the function $P_{s}(x)$ can be written as

$$
P_{s}(x)= \begin{cases}\frac{5}{2} x^{4}+\frac{5}{2} x^{3}-\frac{29}{16} x^{2}-\frac{121}{96} x+\frac{245}{512}, & \text { if } x \in\left[-\frac{3}{4}, \frac{1}{4}\right] . \\ \frac{5}{8} x^{4}+\frac{5}{8} x^{3}-\frac{61}{64} x^{2}-\frac{229}{384} x+\frac{605}{2048}, & \text { if } x \in\left[-1,-\frac{3}{4}\right) \cup\left(\frac{1}{4}, 1\right] .\end{cases}
$$

And its canonical dual function $P^{d}\left(y^{*}\right)$ can be written as

$P^{d}\left(y^{*}\right)$

$$
= \begin{cases}\frac{(-1 / 2)\left(1 / 6-(1 / 4) \epsilon^{*}\right)^{2}}{\left(-1 / 2+\epsilon^{*}+2 \rho^{*}\right)}-\frac{1}{40}\left(\epsilon^{*}\right)^{2}-\frac{7}{32} \epsilon^{*}-\rho^{*}, & \text { if } y^{*} \in\left\{y^{*}=\left(\epsilon^{*}, \rho^{*}\right)^{T} \in R^{2} \mid-\frac{1}{2}+\epsilon^{*}+2 \rho^{*} \neq 0,-5 \leq \epsilon^{*} \leq-\frac{5}{2}, \rho^{*} \geq 0\right\} . \\ \frac{(-1 / 2)\left(1 / 6-(1 / 4) \epsilon^{*}\right)^{2}}{\left(-1 / 2+\epsilon^{*}+2 \rho^{*}\right)}-\frac{3}{32} \epsilon^{*}+\frac{5}{32}-\rho^{*}, & \text { if } y^{*} \in\left\{y^{*}=\left(\epsilon^{*}, \rho^{*}\right)^{T} \in R^{2} \mid-\frac{1}{2}+\epsilon^{*}+2 \rho^{*} \neq 0,-\frac{5}{2}<\epsilon^{*} \leq-\frac{5}{4}, \rho^{*} \geq 0\right\} . \\ \frac{(-1 / 2)\left(1 / 6-(1 / 4) \epsilon^{*}\right)^{2}}{\left(-1 / 2+\epsilon^{*}+2 \rho^{*}\right)}-\frac{1}{10}\left(\epsilon^{*}\right)^{2}-\frac{11}{32} \epsilon^{*}-\rho^{*}, & \text { if } y^{*} \in\left\{y^{*}=\left(\epsilon^{*}, \rho^{*}\right)^{T} \in R^{2} \mid-\frac{1}{2}+\epsilon^{*}+2 \rho^{*} \neq 0, \epsilon^{*}>-\frac{5}{4}, \rho^{*} \geq 0\right\} .\end{cases}
$$




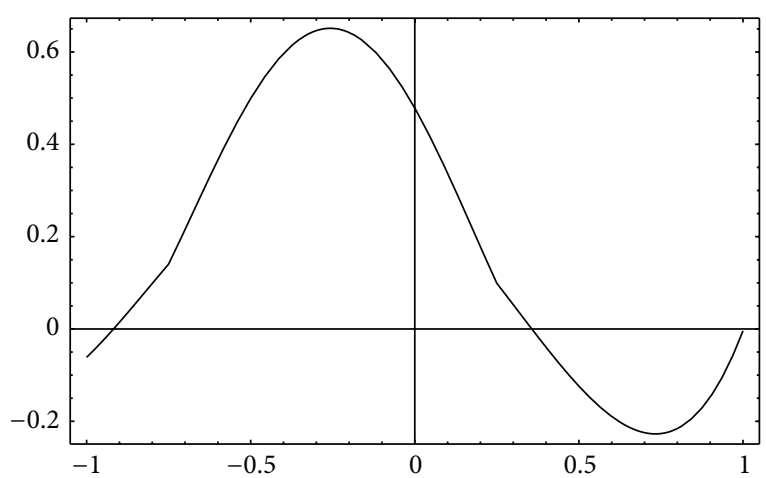

(a)

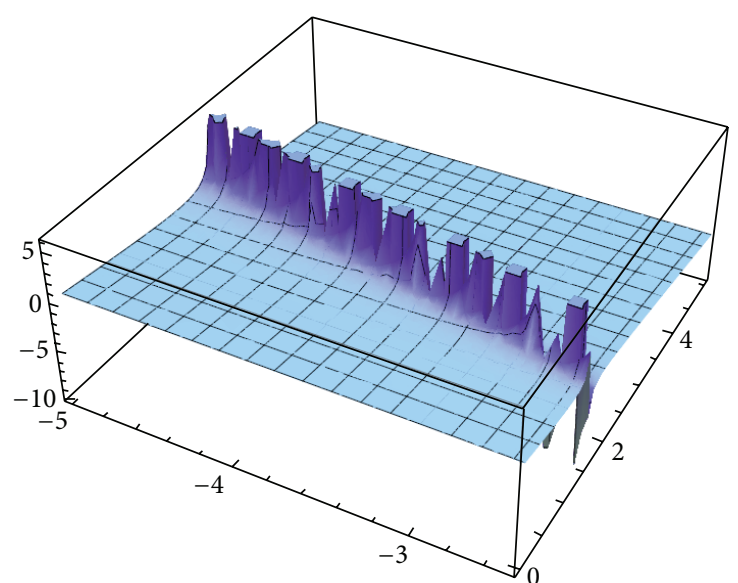

(b)

FIgURE 1: (a) Graph of the $P_{s}(x)$ in one-dimensional spcae. (b) Graph of the canonical dual function $P^{d}\left(y^{*}\right)$.

In the effective domain $\mathscr{Y}_{\alpha_{1}}^{*}=\left\{y^{*}=\left(\epsilon^{*}, \rho^{*}\right)^{T} \in R^{2} \mid-1 / 2+\right.$ $\left.\epsilon^{*}+2 \rho^{*} \neq 0,-5 \leq \epsilon^{*} \leq-5 / 2, \rho^{*} \geq 0\right\}$, the canonical dual function $P^{d}\left(y^{*}\right)$ has one real root:

$$
\bar{y}_{1}^{*}=\left(\bar{\epsilon}_{1}^{*}, \bar{\rho}_{1}^{*}\right)^{T}=(-4.9994,0)^{T} .
$$

In the effective domain $\mathscr{Y}_{\beta_{1}}^{*}=\left\{y^{*}=\left(\epsilon^{*}, \rho^{*}\right)^{T} \in R^{2} \mid-1 / 2+\right.$ $\left.\epsilon^{*}+2 \rho^{*} \neq 0,-5 / 2<\epsilon^{*} \leq-5 / 4, \rho^{*} \geq 0\right\}$, the canonical dual function $P^{d}\left(y^{*}\right)$ does not have any real roots. In the effective domain $\mathscr{Y}_{\gamma_{1}}^{*}=\left\{y^{*}=\left(\epsilon^{*}, \rho^{*}\right)^{T} \in R^{2} \mid-1 / 2+\epsilon^{*}+2 \rho^{*} \neq\right.$ $\left.0, \epsilon^{*}>-5 / 4, \rho^{*} \geq 0\right\}$, the canonical dual function $P^{d}\left(y^{*}\right)$ has two real roots:

$$
\begin{aligned}
& \bar{y}_{2}^{*}=\left(\bar{\epsilon}_{2}^{*}, \bar{\rho}_{2}^{*}\right)^{T}=(-0.4687,0.3424)^{T}, \\
& \bar{y}_{3}^{*}=\left(\bar{\epsilon}_{3}^{*}, \bar{\rho}_{3}^{*}\right)^{T}=(0.5424,0)^{T} .
\end{aligned}
$$

Since $\bar{y}_{3}^{*}=\left(\bar{\epsilon}_{3}^{*}, \bar{\rho}_{3}^{*}\right)^{T} \in \mathcal{Y}_{+}^{*}$, by Theorem 2, we know that

$$
\bar{x}_{3}=\frac{\left(1 / 6-(1 / 4) \bar{\epsilon}_{3}^{*}\right)}{\left(-1 / 2+\bar{\epsilon}_{3}^{*}+2 \bar{\rho}_{3}^{*}\right)}=0.7335
$$

is a global minimizer of $P_{s}(x)$ and $P_{s}\left(\bar{x}_{3}\right)=P^{d}\left(\bar{y}_{3}^{*}\right)=$ $P^{d}\left(\bar{\epsilon}_{3}^{*}, \bar{\rho}_{3}^{*}\right)=-0.2273$, while $\bar{x}_{1}=-0.2576$ is a local maximizer and $\bar{x}_{2}=-1$ is a local minimizer (see Figure 1(a)). The graphs of the box constrained nonconvex and nonsmooth function $P(x)$ and its canonical dual function $P^{d}\left(y^{*}\right)$ are shown in Figure 1.

Example 2. In the case of $n=2$, if we choose $A=B=\left[\begin{array}{ll}1 & 0 \\ 0 & 1\end{array}\right]$, $s=f=\left[\begin{array}{l}1 \\ 1\end{array}\right], 1=a_{1}<a_{2}=9, \mu_{1}=1 / 8, c=1 / 4$, then $\mu_{2}=1 / 16$, and the function $P_{s}(x)$ can be written as

$$
P_{s}(x)= \begin{cases}\frac{1}{2}\left(x_{1}^{2}+x_{2}^{2}-x_{1}-x_{2}\right)+\frac{1}{2}\left[\frac{1}{2}\left(x_{1}-1\right)^{2}+\frac{1}{2}\left(x_{2}-1\right)^{2}-\frac{1}{8}\right]^{2}, & \text { if }\left(x_{1}-1\right)^{2}+\left(x_{2}-1\right)^{2} \leq \frac{1}{16} \\ \frac{1}{2}\left(x_{1}^{2}+x_{2}^{2}-x_{1}-x_{2}\right)+\frac{9}{2}\left[\frac{1}{2}\left(x_{1}-1\right)^{2}+\frac{1}{2}\left(x_{2}-1\right)^{2}-\frac{1}{16}\right]^{2}, & \text { if }\left(x_{1}-1\right)^{2}+\left(x_{2}-1\right)^{2}>\frac{1}{16}\end{cases}
$$

In the effective domain $\mathscr{Y}_{\alpha_{2}}^{*}=\left\{y^{*}=\left(\epsilon^{*}, \rho^{*}\right)^{T} \in R \times R^{2}\right.$ । $\left.1+\epsilon^{*}+2 \rho_{i}^{*} \neq 0,-9 / 16 \leq \epsilon^{*}<-9 / 32, \rho_{i}^{*} \geq 0, i=1,2\right\}$, the canonical dual function $P^{d}\left(y^{*}\right)$ has one real root

$$
\bar{y}_{1}^{*}=\left(\bar{\epsilon}_{1}^{*}, \bar{\rho}_{1}^{*}, \bar{\rho}_{2}^{*}\right)^{T}=\left(-\frac{9}{16}, 0,0\right)^{T} .
$$

Neither in the effective domain $\mathscr{Y}_{\beta_{2}}^{*}=\left\{y^{*}=\left(\epsilon^{*}, \rho^{*}\right)^{T} \epsilon\right.$ $\left.R \times R^{2} \mid 1+\epsilon^{*}+2 \rho_{i}^{*} \neq 0,-9 / 32 \leq \epsilon^{*}<-3 / 32, \rho_{i}^{*} \geq 0, i=1,2\right\}$ nor in the effective domain $\mathcal{Y}_{\gamma_{2}}^{*}=\left\{y^{*}=\left(\epsilon^{*}, \rho^{*}\right)^{T} \in R \times R^{2}\right.$ | $\left.1+\epsilon^{*}+2 \rho_{i}^{*} \neq 0, \epsilon^{*} \geq-3 / 32, \rho_{i}^{*} \geq 0, i=1,2\right\}$, the canonical dual function $P^{d}\left(y^{*}\right)$ have any real roots.
Since $\bar{y}_{1}^{*}=\left(\bar{\epsilon}_{1}^{*}, \bar{\rho}_{1}^{*}, \bar{\rho}_{2}^{*}\right)^{T}=(-9 / 16,0,0)^{T} \in \mathscr{Y}_{+}^{*}$, by Theorem 2, we know that

$$
\bar{x}=F\left(\bar{\epsilon}^{*}, \bar{\rho}^{*}\right)^{-1} G\left(\bar{\epsilon}^{*}\right)=\left[\begin{array}{c}
\frac{1+\epsilon^{*}}{1+\epsilon^{*}+2 \rho_{1}^{*}} \\
\frac{1+\epsilon^{*}}{1+\epsilon^{*}+2 \rho_{2}^{*}}
\end{array}\right]=\left[\begin{array}{l}
1 \\
1
\end{array}\right]
$$

is a global minimizer of $P_{s}(x)$ and $P_{s}(\bar{x})=P^{d}\left(\bar{y}_{1}^{*}\right)=$ $P^{d}\left(\bar{\epsilon}_{1}^{*}, \bar{\rho}_{1}^{*}, \bar{\rho}_{2}^{*}\right)=-1$ (see Figure 2$)$. 


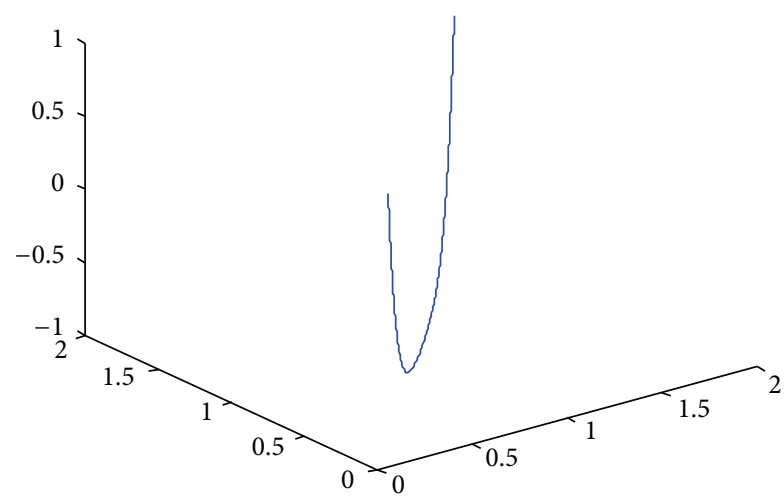

Figure 2: Graph of the function $P_{s}(x)$ in two-dimensional spcae.

\section{Conclusions}

It is very difficult to solve the nonconvex and nonsmooth optimization problem with box constraints via the traditional methods. However, using the canonical dual transformation method, which is presented in the paper, we can easily obtain the global minimizer of $P_{s}(x)$ by solving its canonical dual problem. As the canonical dual function $P^{d}\left(y^{*}\right)$ is concave on the dual feasible space $\mathcal{Y}_{+}^{*}$, when $\mathcal{Y}_{+}^{*}$ is nonempty, the canonical dual function $P^{d}\left(y^{*}\right)$ has one stationary point in $\mathscr{Y}_{+}^{*}$ at least. Thus, we can solve the canonical dual problem by well-developed deterministic optimization methods. The presented paper shows again that the canonical dual transformation method may possess important computational impacts on global optimization.

\section{Conflict of Interests}

The authors declare that there is no conflict of interests regarding the publication of this paper.

\section{Acknowledgments}

This paper was supported by Natural Science Foundation of Guangdong Province (s20120100815) and Youth Foundation of Wuyi University (2013zk14 and 2013zk04).

\section{References}

[1] I. G. Akrotirianakis and C. A. Floudas, "Computational experience with a new class of convex underestimators: boxconstrained NLP problems," Journal of Global Optimization, vol. 29, no. 3, pp. 249-264, 2004.

[2] I. G. Akrotirianakis and C. A. Floudas, "A new class of improved convex underestimators for twice continuously differentiable constrained NLPs," Journal of Global Optimization, vol. 30, no. 4, pp. 367-390, 2004.

[3] C. A. Floudas, Deterministic Optimization. Theory, Methods, and Applications, Kluwer Academic Publishers, 2000.

[4] C. A. Floudas, I. G. Akrotirianakis, S. Caratzoulas, C. A. Meyer, and J. Kallrath, "Global optimization in the 21st century: advances and challenges," Computers and Chemical Engineering, vol. 29, no. 6, pp. 1185-1202, 2005.

[5] L. Q. Qi and J. Sun, "A trust region algorithm for minimization of locally Lipschitzian functions," Mathematical Programming, vol. 66, pp. 25-43, 1994.

[6] H. D. Sherali and A. Alameddine, "A new reformulationlinearization technique for bilinear programming problems," Journal of Global Optimization, vol. 2, no. 4, pp. 379-410, 1992.

[7] B. C. Shi and X. S. Hu, "Maximum entropy method and nonmonotone curvlinear search method for unconstrained optimization," Mathematica Numerica Sinica, vol. 19, no. 3, pp. 241-256, 1997 (Chinese).

[8] Y. Yuan, "Conditions for convergence of trust region algorithms for nonsmooth optimization," Mathematical Programming, vol. 31, no. 2, pp. 220-228, 1985.

[9] X. J. Zhou, D. Y. Gao, and C. H. Yang, “Canonical primal-dual algorithm for solving fourth-order polynomial minimization problems," Applied Mathematics and Computation, vol. 227, pp. 246-255, 2014.

[10] J. Liu, D. Y. Gao, and Y. Gao, "Perfect duality theory and solutions to a class of unconstrained nonconvex and nonsmooth optimization," Optimization and Engineering, vol. 108, no. 3, pp. 158-167, 2009.

[11] F. Falk, "Elastic phase transitions and nonconvex energy functions," in Free Boundary Problem: Theory and Applications I, K. H. Hoffmann and J. Sprekels, Eds., vol. 185, pp. 45-59, Longman, London, UK, 1990.

[12] A. Rubinov and X. Yang, Lagrange-Type Functions in ConstrainedNon-Convex Optimization, vol. 85 of Applied Optimization, Kluwer Academic Publishers, Boston, Mass, USA, 2003.

[13] A. A. Atai and D. J. Steigmann, "Coupled deformations of elastic curves and surfaces," International Journal of Solids and Structures, vol. 35, no. 16, pp. 1915-1952, 1998.

[14] D. Dacorogna, Direct Methods in the Calculus of Variations, Springer, 2008.

[15] I. Ekeland, "Legendre duality in nonconvex optimization and calculus of variations," SIAM Journal on Control and Optimization, vol. 15, no. 6, pp. 905-934, 1977.

[16] R. N. Gasimov, "Augmented Lagrangian duality and nondifferentiable optimization methods in nonconvex programming," Journal of Global Optimization, vol. 24, no. 2, pp. 187-203, 2002.

[17] A. M. Rubinov, X. Q. Yang, and B. M. Glover, "Extended Lagrange and penalty functions in optimization," Journal of Optimization Theory and Applications, vol. 111, no. 2, pp. 381405, 2001.

[18] P. T. Thach, H. Konno, and D. Yokota, "Dual approach to minimization on the set of Pareto-optimal solutions," Journal of Optimization Theory and Applications, vol. 88, no. 3, pp. 689$707,1996$.

[19] M. H. Wright, "The interior-point revolution in constrained optimization," in High Performance Algorithms and Software in Nonlinear Optimization, R. Deleone, A. Murli, P. M. Pardalos, and G. Toraldo, Eds., pp. 359-381, Kluwer Academic, Dodrecht, The Netherlands, 1998.

[20] Y. Ye, "A new complexity result on minimization of a quadratic function with a sphere constraint," in Recent Advances in Glibal Optimization, C. Floudas and P. Pardalos, Eds., pp. 19-31, Princeten University Press, Princeton, NJ, USA, 1992. 
[21] D. Y. Gao, "Perfect duality theory and complete solutions to a class of global optimization problems," Optimization, vol. 52, no. 4-5, pp. 467-493, 2003.

[22] D. Y. Gao, "Canonical duality theory and solutions to constrained nonconvex quadratic programming," Journal of Global Optimization, vol. 29, no. 4, pp. 377-399, 2004. 


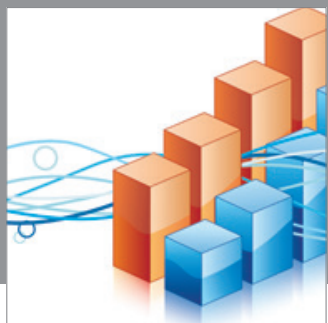

Advances in

Operations Research

mansans

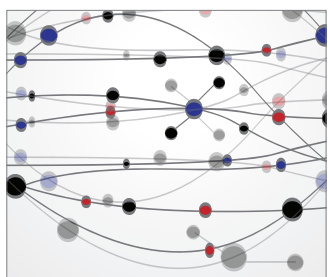

The Scientific World Journal
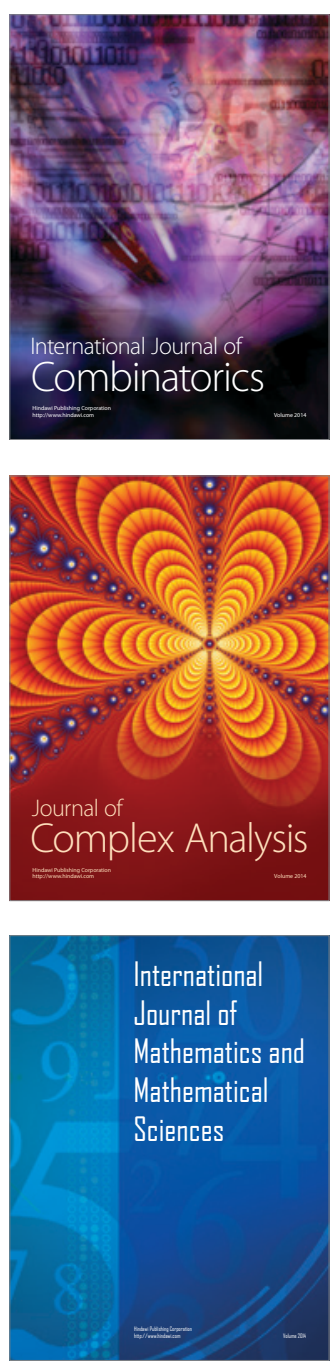
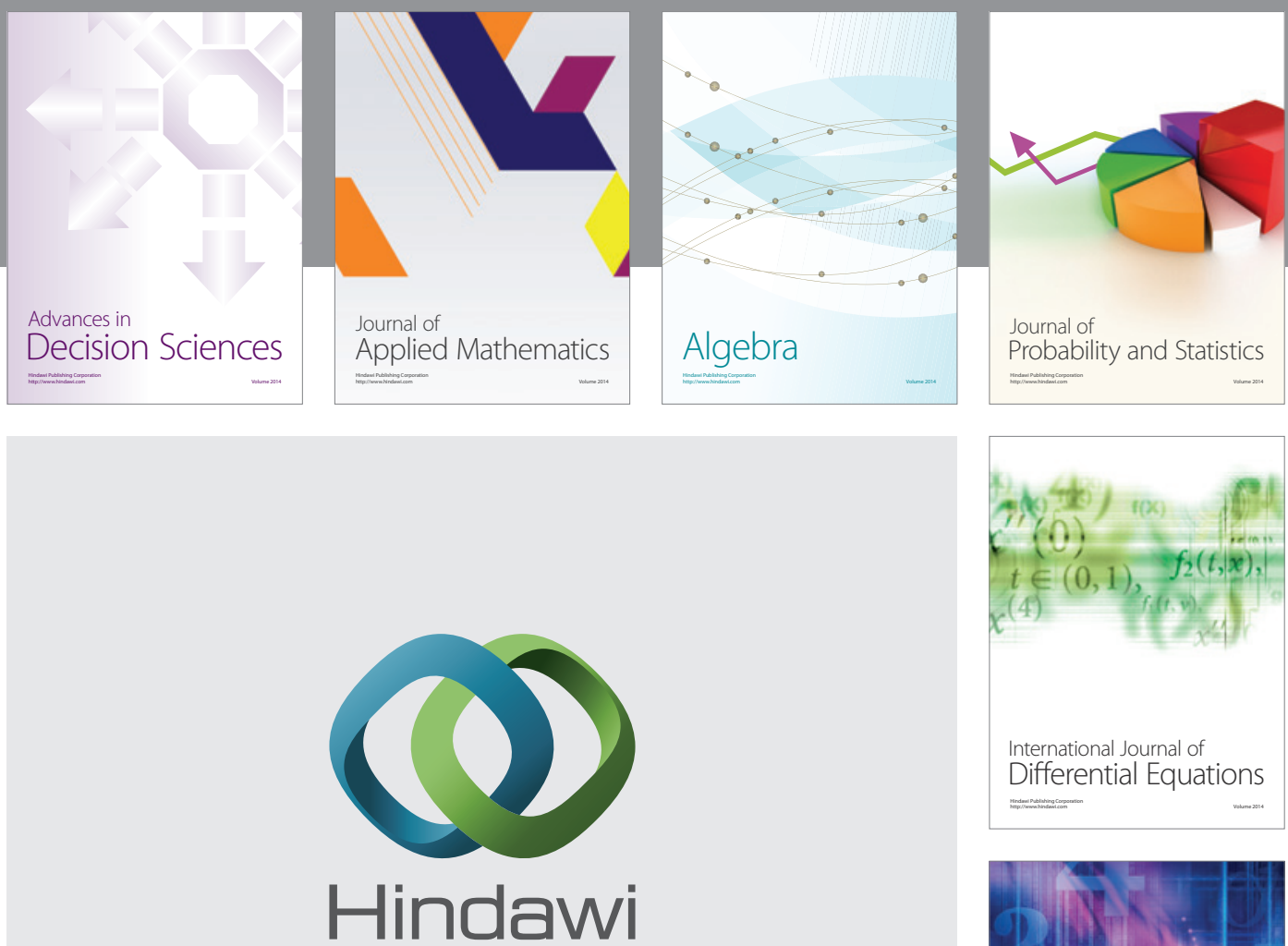

Submit your manuscripts at http://www.hindawi.com
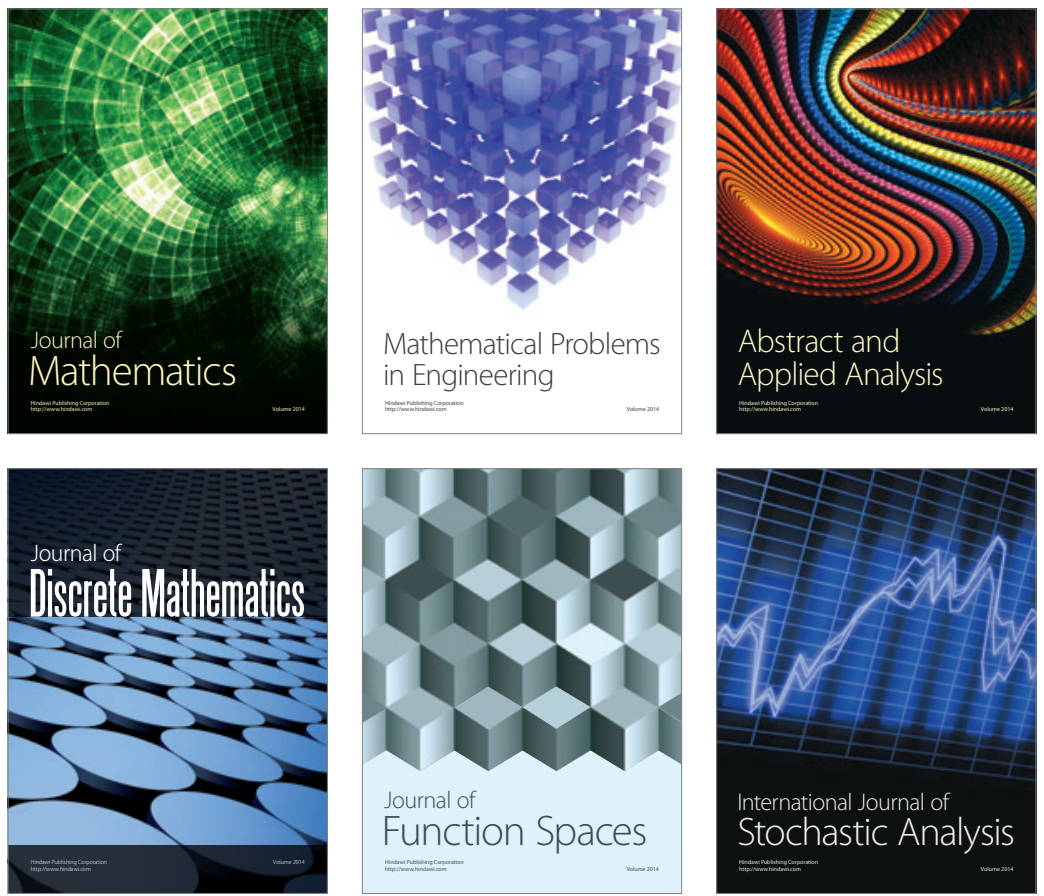

Journal of

Function Spaces

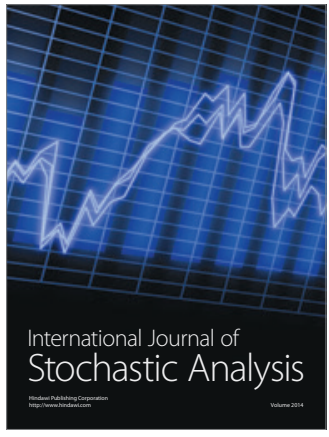

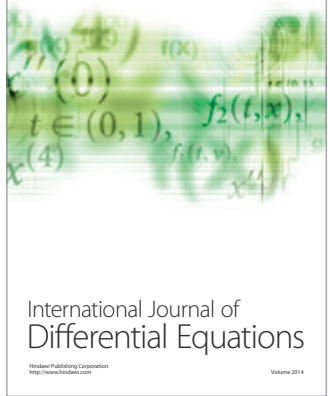
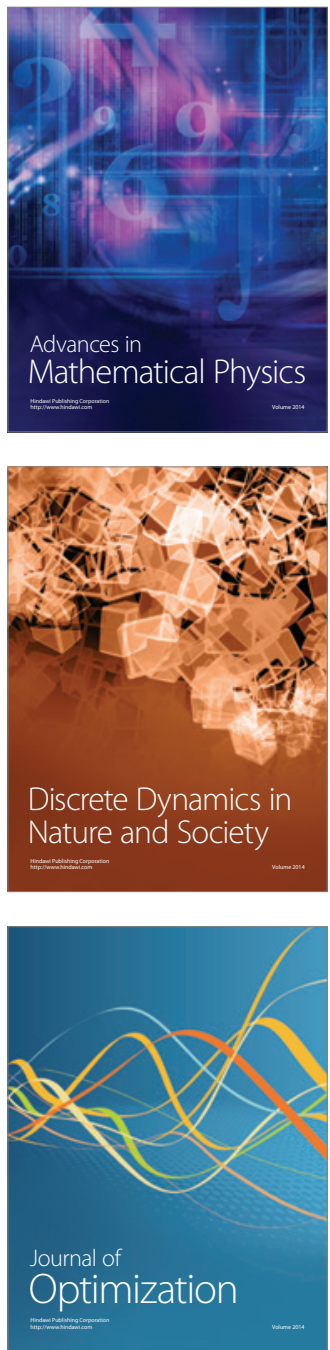